\title{
A physiological approach for pre-selection of Eucalyptus clones resistant to drought
}

\author{
Caroline Müller ${ }^{(1-2)}$, \\ Bárbara Elias Reis Hodecker ${ }^{(1-2)}$, \\ Nairam Félix De Barros ${ }^{(1)}$, \\ Andrew Merchant ${ }^{(2)}$
}

\begin{abstract}
Water deficit is one of the abiotic stresses that most affects the growth and survival of Eucalyptus. Mechanisms used to tolerate water-limited environments influence the distribution of Eucalyptus species in their natural environment. Here, we take a physiological approach to pre-screen Eucalyptus plants for tolerance to drought. Ten different clones of $E$. urophylla and $E$. grandis $\times E$. urophylla that are known to show contrasting responses to water deficit under field conditions, were grown in Clark's nutrient solution (WW, well-watered) and with polyethylene glycol $(-1.0 \mathrm{MPa})$ to simulate water deficit (WD). Clones responded differently to drought with differentiated photosynthetic limitations in drought-treated clones. Photosynthetic rates, stomatal conductance, transpiration and internal $\mathrm{CO}_{2}$ concentrations were reduced in all genotypes under stress conditions. Clone $\mathrm{i} 144$ had a smaller reduction in the evaluated physiological traits, also showing increased root growth in WDtreated plants. Clones 3367 and i224, thought to be moderately tolerant, also followed these patterns. Clones gg157, 1568 and 1641, all of which are moderately sensitive under field conditions, reduced most of the physiological characters evaluated. However, clone gg157 demonstrated increased root system growth, even during short periods of water stress. Clones i042 and i182 were deemed drought-susceptible, with large reductions in photosynthesis and growth, despite showing a high increase in abscisic acid content presumably as a defense mechanism. Interaction between $A$ (photosyntetic rate), $E$ (transpiration rate), ETR/A (electrons transport rate/photosynthetic rate) and SDM/ RDM (shoot dry matter/root dry matter) demonstrated the most significant differences between WD-treated clones and offer great potential for use as selection criterion for water deficit-tolerant genotypes.
\end{abstract}

\section{Keywords: Clonal Variability, Genotypes, Cluster Analysis, Water Stress}

ferential characteristic among species of the genus (Merchant et al. 2007, Zhou et al. 2016). Therefore, understanding the physiological, biochemical and hormonal responses to drought in various Eucalyptus genotypes is fundamental to select drought-tolerant clones to be cultivated in environments affected by water shortage (White et al. 2009).

Several mechanisms may participate in responses to water deficit, including processes that limit dehydration maintaining higher water potential, and/or processes that allow tissues to tolerate lower water
(1) Department of Soil Science, Federal University of Viçosa, Viçosa MG, 36571-000 (Brazil); (2) School of Life and Environmental Sciences, Centre for Carbon, Water and Food, The University of Sydney, Camden NSW, 2570 (Australia)

@ Caroline Müller (carolinemulleram@gmail.com)

Received: Jul 04, 2019 - Accepted: Nov 21, 2019

Citation: Müller C, Hodecker BER, De Barros NF, Merchant A (2020). A physiological approach for pre-selection of Eucalyptus clones resistant to drought. iForest 13: 16-23. - doi: 10.3832/ifor3185-012 [online 2020-01-15]

Communicated by: Claudia Cocozza potential. In the first case, plants use strategies that minimize water loss through regulation of transpiration via stomatal control (Macfarlane et al. 2004). Photosynthesis is the physiological process most affected by water stress (Warren et al. 2007). Under these circumstances, plants induce hormone-mediated responses, including those governed by abscisic acid (ABA), often also improving water-use efficiency, thereby regulating plant-scale adaptive responses to restrictive conditions (Schachtman \& Goodger 2008).

Under severe and long-term drought, reduction in net photosynthetic rate may also be the result of biochemical limitations in photosynthetic metabolism, including limitations in phosphorylation (Lawlor \& Tezara 2009), ribulose-1,5-bisphosphate (RuBP) regeneration (Medrano et al. 2002) and RuBisCO carboxylation (Zhou et al. 2007). Both processes favor the reduction of carbon assimilation by plants and lead to super-excitation and accumulation of reducing power in the leaves. Protective mechanisms against excess reducing power are important strategies to combat drought-stress; they should therefore also be investigated to obtain a better understanding of drought tolerance by plants. 
Drought stress elicits a range of concomitant effects that may limit growth and threaten survival, including photon flux in excess of that required for photoassimilation. To cope with these conditions, protective mechanisms possessed by drought-tolerant plants generally compete with the photochemical phase for absorbed energy, leading to a decrease in PS II quantum yield (Genty et al. 1989) and increasing thermal dissipation (Demming-Adams \& Adams 1996). These findings suggest that thermal dissipation helps to protect against oxidative damage and photoinhibition (Murata et al. 2012). Simultaneous measurement of gas exchange and chlorophyll a fluorescence is therefore an important tool for understanding the relationship between the use-efficiency of light, $\mathrm{CO}_{2}$ assimilation and photoinhibition, in addition to being an important indicator of water deficit in plants (Maxwell \& Johnson 2000).

Several studies have been conducted to identify the physiological and morphological modifications caused by low water availability in Eucalyptus. Modifications such as reduced photosynthetic rate (Warren et al. 2011), changes in metabolite production (Merchant et al. 2006, Warren et al. 2012), growth, and productivity (Macfarlane et al. 2004, Merchant et al. 2010) are the most common outcomes. Although many drought tolerance mechanisms have been studied, forest companies release new, relatively untested clones in each growth season with clone selection constrained by end-user specifications for timber quality. A fast primary screening for water deficit tolerance at the seedling stage assists identification and selection of superior genotypes to be taken to the field. In addition, it is noteworthy that each genotype has its own genetic characteristics and in general, several experiments have been only focused in physiological comparison between two contrasting genotypes. These make it more difficult to evaluate whether the analyses taken were suitable for pre-selection of drought tolerant genotypes.

Recently, early selection has been carried out in several Eucalyptus species. However, few studies have sought to complete a concurrent screen of multiple traits to investigate interactive and correlative properties of candidate characteristics contributing to water deficit tolerance (Pinto et al. 2014, Corrêa et al. 2017). Therefore, the purpose of the present study was to evaluate morphological, physiological and noninvasive traits that could potentially discriminate amongst clones for tolerance to water deficit. Using a fast aquaponics methodology on young seedlings, our objective was to provide quantitative physiological markers for initial screening of ten juvenile eucalypt different clones for their tolerance to the effects of water deficit.

\section{Material and methods}

Plant material and experimental design

Seedlings of ten Eucalyptus clones ( $E$. grandis $\times$ E. urophylla hybrid: 1528,3367 and VC865; Eucalyptus urophylla hybrid: clones 1641, gg157, i042, i144, i182, i224 and PL40) were used for the experiment. Clones i042 and 1144 were previously characterized by the research group as drought-sensitive and -tolerant, respectively, and were used as reference for comparison with other genotypes in this study. Based on the previous observations, categorizations of sensitivity adopted throughout the manuscript are relative (not absolute). Terms adopted throughout this manuscript are therefore used in this context. Clones i042 and i144 were used in this study to ensure that a broad scope of field performance was reflected in the seedlings screened. Plants were grown in a greenhouse in plastic pots $(8 \mathrm{~L})$ and were acclimated in Clark nutrient solution (Clark 1975). After 30 days of acclimation, the plants were exposed to two different water treatments: nutrient solution with water potential near o MPa (WW, well-watered) and subjected to water deficit (WD), initiated by the gradual addition of polyethylene glycol (PEG) 6000 (Vetec Quimica Fina LTDA, RJ, Brazil) to the nutrient solution. PEG 6000 doses were added every five days to gradually reduce the water potential of the solution (to -0.16, -0.32, -0.65, and -1.0 MPa - Michel \& Kaufmann 1973). The experiment was arranged in a randomized block, using a $10 \times 2$ factorial design scheme (genotypes and water availability, respectively), with four biological replications and two plants per pot.

\section{Water potential and gas exchange} traits

Five days after application of a PEG 6000 dose to reduce the water potential of the solution to $-1 \mathrm{MPa}$, predawn leaf water potential was measured using a pressure chamber (Scholander, Corvallis, USA) in order to evaluate the effective water deficiency imposed by PEG 6000.

Net photosynthetic assimilation rate $(A)$, stomatal conductance $\left(g_{s}\right)$, transpiration rate $(E)$, and the ratio between internal and external $\mathrm{CO}_{2}$ concentration $\left(C_{i} / C_{a}\right)$ were measured using an infrared gas analyzer (IRGA ${ }^{\circledast}$, LI-6400xt, LI-COR Bioscience Inc., Lincoln, NE, US). Measurements were performed in fully-expanded leaves, between 8:00 am and 12:30 pm, under constant photosynthetically active radiation (PAR, 1400 $\mu$ mol photons $\left.\mathrm{m}^{-2} \mathrm{~s}^{-1}\right)$, atmospheric $\mathrm{CO}_{2}$ concentration $\left(C_{a}\right)$ of $\sim 372 \mu \mathrm{mol} \mathrm{mol}^{-1}$, at 22-28 ${ }^{\circ} \mathrm{C}$ and $48-66 \%$ relative humidity. Carboxylation efficiency was calculated using $A$ and $C_{i}$ values $\left(A / C_{i}\right)$ and the intrinsic water use efficiency (WUE) was calculated as the ratio between $A$ and $g_{s}$.

\section{Chlorophyll a fluorescence}

Variables of chlorophyll a fluorescence were measured using a fluorometer cou- pled to an IRGA (LI-6400xt ${ }^{\oplus}$, Li-Cor Biosciences, NE, USA). Leaves were initially dark-adapted to obtain the initial fluorescence $\left(F_{0}\right)$ and maximal fluorescence $\left(F_{m}\right)$. From this data, the potential quantum yield of photosystem II (PSII; $\left.F_{v} / F_{m}\right)$ was obtained. Leaf tissues were then exposed to actinic light and a saturating pulse to obtain the steady-state fluorescence (F) and the maximum fluorescence in a lightadapted state $\left(\mathrm{F}_{\mathrm{m}}{ }^{\prime}\right)$, respectively. This allowed determination the minimum fluorescence of the illuminated plant tissue $\left(\mathrm{F}_{\circ}{ }^{\prime}\right)$, the coefficient photochemical quenching $(\mathrm{qL})$, the effective quantum yield of photochemical energy conversion in PSII $\left(\mathrm{Y}_{\|}\right)$, the quantum yield of non-regulated energy $\left(\mathrm{Y}_{\mathrm{NPQ}}\right)$ and the apparent electron transport rate (ETR), as detailed by Müller et al. (2017).

\section{Quantification of abscisic acid (ABA)}

$A B A$ was extracted from ground frozen leaf material (100 $\mathrm{mg}$ ) using methanol $(80 \%, 1 \mathrm{~mL})$ as extraction solvent, according to Durgbanshi et al. (2005), with modifications. Two hundred microliters of the sample extract had the methanol removed in a SpeedVac at $30{ }^{\circ} \mathrm{C}$ and the sample was resuspended in water with the $\mathrm{pH}$ corrected to 3.0 (100 $\mu \mathrm{l}$ of $10 \% \mathrm{v} / \mathrm{v}$ acetic acid). Diethyl-ether $(500 \mu \mathrm{l})$ was added to allow phase separation. The organic phase was collected in a tube and the extract was washed again in diethyl-ether, repeating the process. The extract was dried using a heat block and the samples dissolved in LCMS buffer for analysis. Leaf extracts containing $A B A$ were analyzed using a Triple Quadrupole LC-MS $6430^{\circledR}$ (Agilent Technologies, Santa Clara, CA, USA) with the following settings: column (Agilent Eclipse Plus $^{\oplus}$, RRHD, $1.8 \mu \mathrm{m}, 2.1 \times 50 \mathrm{~mm}$ with guard column), solvents (A: Acetonitrile + $0.1 \%$ formic acid, B: Water $+0.1 \%$ formic acid), flow $\left(0.3 \mathrm{~mL} \mathrm{~min}^{-1}\right.$ ) for $7 \mathrm{~min}$ (gradient time/B\%: o/81, 3/50, 4/10, 4.25/10, 4.5/81). Deuterated ABA (ABAd4) standard was added during the extraction process. Detection and quantification of $A B A$ in the samples was made by multiple reaction monitoring (MRM) by means of selecting the transition density of the molecule of interest (ABA $263 \rightarrow 153$; ABAd4 $267 \rightarrow 156$ ), using the following Mass spectrometric parameters: Dwell 200, Fragmentor 60, CE 6, Accelerator voltage 7, Negative polarity. Data were processed using the software MassHunter workstation VB 06.00 (Agilent Technologies).

\section{Chloroplast pigments}

Pigments were extracted from leaf discs $\left(0.6 \mathrm{~cm}^{2}\right)$ using dimethyl sulfoxide $(5 \mathrm{~mL})$ saturated with calcium carbonate, under dark conditions at $65^{\circ} \mathrm{C}$. After four hours, absorbances were measured at 480, 649.1 at $665.1 \mathrm{~nm}$ in a spectrophotometer. Pure DMSO was used as a blank. Absorbance values were used to estimate chlorophyll a (Chla), chlorophyll b (Chlb) and total ca- 
rotenoids (Carot) as described by Wellburn (1994). Pigment concentration was expressed on the basis of leaf area.

\section{Natural abundance carbon isotope composition $\left(\delta^{13} \mathrm{C}\right)$}

The isotopic composition was analyzed in fully expanded-leaves for abundance of ${ }^{13} \mathrm{C}$ $\left(\delta^{13} \mathrm{C}, \%\right)$, with an isotope ratio mass spectrometer (IRMS, Delta $\mathrm{V}$ Plus ${ }^{\circledR}$ model, Thermo Scientific, Waltham, MA, USA). The relationship between the stable isotopes of $C$ was calculated by the formula: $\delta^{13} \mathrm{C}=$ $\left(R_{\text {sample }} / R_{\text {standard }}\right)-1$, where $R$ is the molar ratio of the heavy $\left({ }^{13} \mathrm{C}\right)$ and light isotopes $\left({ }^{12} \mathrm{C}\right)$. The isotope discrimination was calculated by (eqn. 1):

$$
\Delta=\frac{\delta^{13} C_{a t m}-\delta^{13} C_{\text {plant }}}{1+\delta^{13} C_{\text {plant }}}
$$

where $\delta^{13} C_{a t m}$ corresponded to $8 \%$ (Farquhar \& Richards 1984).

\section{Morphological traits}

The height $(\mathrm{cm})$ and stem diameter $(\mathrm{mm})$ of the plants were measured using a ruler and a caliper, respectively. Leaves, stem and roots were wrapped in paper bags, oven-dried in a forced air circulation oven $\left(65^{\circ} \mathrm{C}\right)$ for $72 \mathrm{~h}$ and weighed to determine the shoot dry matter (SDM), root dry matter (RDM), total dry matter (TDM) and the shoot and root dry matter ratio (SDM/ RDM) and root and total dry matter ratio (RDM/TDM).

\section{Statistical analysis}

Data were subjected to factorial ANOVA using Scott-Knott clustering $(p<0.01$ and $p$
$<0.05)$. The relationships between variables were evaluated using principal component analysis (PCA). The relative data for PCA and hierarchical clustering analysis were obtained from the normalization of the original data set as log of the ratio between the water stress treatment and the control values. Clones were grouped according to the tolerant or sensitive characters by the dendrogram using Ward's linkage with Bray-Curtis dissimilarity measure. All statistical analysis were developed using the "vegan" package implemented in the $R$ software ver. 3.2.0 ( $R$ Development Core Team, AKL, New Zealand).

\section{Results}

Water relations and gas exchange traits A gradual increase in PEG concentration in the hydroponic solutions resulted in lower predawn leaf water potentials $\left(\Psi_{w}\right)$, suggesting that plants were under water stress. The WW and WD plants showed $\Psi_{w}$ an average of -0.26 and $-1.35 \mathrm{MPa}$, respectively (Tab. 1). The net photosynthetic assimilation rate $(A)$ of drought-stressed Eucalyptus plants was affected in all genotypes ( $p<0.05-$ Tab. 1$)$. The largest percentage reductions in $A$ were observed for clones i042 (4.02 $\left.\mu \mathrm{mol} \mathrm{m} \mathrm{m}^{-2} \mathrm{~s}^{-1}, 82 \%\right)$, gg157 $\left(5.94 \mu \mathrm{mol} \mathrm{m} \mathrm{m}^{-2} \mathrm{~s}^{-1}, 75 \%\right)$ and $\mathrm{i} 182(7.45 \mu \mathrm{mol}$ $\left.\mathrm{m}^{-2} \mathrm{~s}^{-1}, 63 \%\right)$ under drought stress compared to plants under normal water availability. In clones i144, 3367 and i244, the reduction of $A, 31 \%\left(12.72 \mu \mathrm{mol} \mathrm{m} \mathrm{m}^{-2} \mathrm{~s}^{-1}\right), 40 \%(10.97$ $\left.\mu \mathrm{mol} \mathrm{m} \mathrm{m}^{-1}\right)$ and $48 \%\left(9.17 \mu \mathrm{mol} \mathrm{m} \mathrm{m}^{-2} \mathrm{~s}^{-1}\right)$, respectively, was less pronounced when the plants were subjected to WD treatment, compared to WW plants. Similarly, water deficit caused a reduction in stomatal conductance $\left(g_{s}\right)$ and transpiration rate $(E)$. The carboxylation efficiency $\left(A / C_{i}\right)$ was reduced in all clones under WD, except for clone i144 (Tab. 1) and the water use efficiency (WUE) increased in all clones up to three-fold in io $42\left(110.7 \mu \mathrm{mol} \mathrm{CO}_{2} \mathrm{~mol}^{-1} \mathrm{H}_{2} \mathrm{O}\right)$ and $1182\left(118.8 \mu \mathrm{mol} \mathrm{CO} \mathrm{mol}^{-1} \mathrm{H}_{2} \mathrm{O}\right)$ in WD plants compared to WW treatment (Tab. 1).

\section{Chlorophyll a fluorescence}

Chlorophyll a fluorescence was affected by water stress and genotype $(p<0.05)$. Initial chlorophyll a fluorescence $\left(F_{0}-\right.$ data not shown) and the potential quantum efficiency of PSII $\left(F_{v} / F_{m}\right)$ showed minor variations in WD plants (Tab. 2). Responses in the electron transport rate (ETR - data not shown) and the ratio ETR/A were reduced dramatically by the onset of water deficit, with the largest reduction for clones i042 (77\%, 0.04) and $1182(60 \%, 0.006)$ and the lowest for clone i144 $(28 \%, 0.010)$ compared to WW plants ( $\mathrm{p}<0.05-$ Tab. 2$)$. The estimate of PSII open centers ( $q \mathrm{~L}$ ) increased significantly ( $<<0.05)$ in clone 1144 $(45 \%, 0.08)$ and decreased in io42 (42\%, 0.049) compared to well-watered plants (Tab. 2). The quantum yield of non-regulated energy $\left(\mathrm{Y}_{\mathrm{NPQ}}\right)$ of WD plants, increased around $25 \%$ for the clones gg157 (0.55), io 42 (0.59), 1528 (0.62), 1641 (0.59) and i182 (0.58), compared to WW plants ( $\mathrm{p}<0.05-$ Tab. 2).

\section{ABA, pigment content and isotopic discrimination}

Water deficit increased ABA levels in the

Tab. 1 - Predawn water potential $\left(\Psi_{w},-\mathrm{MPa}\right)$; net photosynthetic assimilation rate $\left(A, \mu \mathrm{mol} \mathrm{CO}_{2} \mathrm{~m}^{-2} \mathrm{~s}^{-1}\right)$; stomatal conductance $\left(\mathrm{g}_{\mathrm{s}}\right.$, mol $\left.\mathrm{H}_{2} \mathrm{O} \mathrm{m}^{-2} \mathrm{~s}^{-1}\right)$; transpiration rate $\left(E, \mathrm{mmol} \mathrm{H}_{2} \mathrm{O} \mathrm{m}^{-2} \mathrm{~s}^{-1}\right)$, carboxylation efficiency $\left(A / C_{i}\right)$ and intrinsic water use efficiency $\left(\right.$ WUE, $\mu$ mol $\mathrm{CO}_{2}$ $\mathrm{mol}^{-1} \mathrm{H}_{2} \mathrm{O}$ ) in Eucalyptus clones grown in well-watered (WW) or water deficit (WD) conditions. Data are presented as the mean $\pm \mathrm{SE}$ $(n=4)$. Capital letters compare different clones under well-watered $(W W)$ treatment, and lower letters compare different clones under water deficit (WD) treatment using the Scott-Knott clustering ( $\mathrm{p}<0.05)$. Asterisk compares WW and WD treatments within each clone. $\left({ }^{* *}\right)$ : $p<0.01 ;(*): p<0.05$.

\begin{tabular}{|c|c|c|c|c|c|c|c|}
\hline Clone & Treat & $\Psi_{\mathrm{w}}$ & $A$ & $g_{s}$ & $E$ & $A / C_{\mathrm{i}}$ & WUE \\
\hline \multirow[t]{2}{*}{1528} & WW & $0.26 \pm 0.02 \mathrm{~A}$ & $18.96 \pm 2.03 \mathrm{~A}$ & $0.379 \pm 0.06 \mathrm{~B}$ & $5.08 \pm 0.77 B$ & $0.071 \pm 0.005 \mathrm{~A}$ & $52.7 \pm 5.3 \mathrm{~A}$ \\
\hline & WD & $1.58 \pm 0.13 a^{* *}$ & $6.36 \pm 0.88 b^{* *}$ & $0.063 \pm 0.01 a^{* *}$ & $1.28 \pm 0.20 a^{*}$ & $0.032 \pm 0.004 b$ & $100.8 \pm 8.3 a^{* *}$ \\
\hline \multirow[t]{2}{*}{1641} & WW & $0.20 \pm 0.04 B$ & $21.27 \pm 1.46 \mathrm{~A}$ & $0.354 \pm 0.08 B$ & $4.90 \pm 0.73 B$ & $0.087 \pm 0.012 \mathrm{~A}$ & $81.6 \pm 33.9 \mathrm{~A}$ \\
\hline & WD & $1.25 \pm 0.09 c^{* *}$ & $9.29 \pm 0.85 a^{* *}$ & $0.085 \pm 0.01 a^{* *}$ & $1.59 \pm 0.08 a^{* *}$ & $0.050 \pm 0.006 a$ & $109.1 \pm 4.1 \mathrm{a}$ \\
\hline \multirow[t]{2}{*}{367} & WW & $0.25 \pm 0.08 \mathrm{~A}$ & $18.40 \pm 0.66 \mathrm{~A}$ & $0.384 \pm 0.06 \mathrm{~B}$ & $4.97 \pm 0.53 B$ & $0.069 \pm 0.006 \mathrm{~A}$ & $51.7 \pm 8.3 \mathrm{~A}$ \\
\hline & WD & $1.38 \pm 0.22 c^{* *}$ & $10.97 \pm 1.80 b^{* *}$ & $0.120 \pm 0.02 a^{* *}$ & $2.14 \pm 0.24 a^{* *}$ & $0.051 \pm 0.007 a$ & $91.2 \pm 1.6 a^{* *}$ \\
\hline \multirow[t]{2}{*}{ gg157 } & WW & $0.15 \pm 0.07 B$ & $23.61 \pm 0.79 \mathrm{~A}$ & $0.502 \pm 0.04 \mathrm{~A}$ & $5.86 \pm 0.27 \mathrm{~A}$ & $0.087 \pm 0.002 \mathrm{~A}$ & $47.7 \pm 3.3 \mathrm{~A}$ \\
\hline & WD & $0.96 \pm 0.16 b^{* *}$ & $5.94 \pm 1.00 b^{* *}$ & $0.063 \pm 0.01 a^{* *}$ & $1.23 \pm 0.13 a^{* *}$ & $0.030 \pm 0.008 b$ & $92.5 \pm 9.1 \mathrm{a}^{* *}$ \\
\hline \multirow[t]{2}{*}{ i042 } & WW & $0.18 \pm 0.09 \mathrm{~A}$ & $21.36 \pm 1.27 \mathrm{~A}$ & $0.543 \pm 0.03 \mathrm{~A}$ & $6.25 \pm 0.39 \mathrm{~A}$ & $0.076 \pm 0.007 \mathrm{~A}$ & $39.7 \pm 2.8 \mathrm{~A}$ \\
\hline & WD & $1.59 \pm 0.07 c^{* *}$ & $4.02 \pm 0.37 b^{* *}$ & $0.036 \pm 0.00 a^{* *}$ & $0.75 \pm 0.06 a^{* *}$ & $0.023 \pm 0.005 b$ & $110.7 \pm 11.7 a^{* *}$ \\
\hline \multirow[t]{2}{*}{ ¡144 } & WW & $0.30 \pm 0.12 \mathrm{~A}$ & $18.39 \pm 0.53 \mathrm{~A}$ & $0.506 \pm 0.01 \mathrm{~A}$ & $6.58 \pm 0.28 \mathrm{~A}$ & $0.062 \pm 0.002 \mathrm{~A}$ & $36.4 \pm 1.3 \mathrm{~A}$ \\
\hline & WD & $1.27 \pm 0.10 b^{* *}$ & $12.72 \pm 2.15 a^{* *}$ & $0.140 \pm 0.03 a^{* *}$ & $2.81 \pm 0.52 a^{* *}$ & $0.063 \pm 0.010 a$ & $93.4 \pm 5.0 a^{* *}$ \\
\hline \multirow[t]{2}{*}{ i182 } & WW & $0.44 \pm 0.06 B$ & $20.16 \pm 0.85 \mathrm{~A}$ & $0.546 \pm 0.03 \mathrm{~A}$ & $6.38 \pm 0.43 \mathrm{~A}$ & $0.070 \pm 0.004 \mathrm{~A}$ & $37.4 \pm 3.06 \mathrm{~A}$ \\
\hline & WD & $1.53 \pm 0.14 c^{* *}$ & $7.45 \pm 0.90 b^{* *}$ & $0.067 \pm 0.01 a^{* *}$ & $1.34 \pm 0.12 a^{* *}$ & $0.043 \pm 0.007 a$ & $111.8 \pm 11.5 a^{* *}$ \\
\hline \multirow[t]{2}{*}{1224} & WW & $0.30 \pm 0.06 \mathrm{~A}$ & $17.75 \pm 2.03 \mathrm{~A}$ & $0.376 \pm 0.08 \mathrm{~B}$ & $4.55 \pm 0.78 B$ & $0.066 \pm 0.005 \mathrm{~A}$ & $56.7 \pm 14.5 \mathrm{~A}$ \\
\hline & WD & $1.23 \pm 0.03 c^{*}$ & $9.17 \pm 1.04 a^{* *}$ & $0.102 \pm 0.01 a^{* *}$ & $2.02 \pm 0.30 a^{* *}$ & $0.044 \pm 0.007 a$ & $90.7 \pm 6.9 a^{* *}$ \\
\hline \multirow[t]{2}{*}{ PL40 } & WW & $0.36 \pm 0.04 \mathrm{~B}$ & $19.80 \pm 1.06 \mathrm{~A}$ & $0.360 \pm 0.07 \mathrm{~B}$ & $4.88 \pm 0.64 B$ & $0.078 \pm 0.003 \mathrm{~A}$ & $59.8 \pm 8.5 \mathrm{~A}$ \\
\hline & WD & $1.36 \pm 0.12 c^{* *}$ & $8.28 \pm 1.09 b^{* *}$ & $0.071 \pm 0.01 a^{* *}$ & $1.28 \pm 0.14 a^{* *}$ & $0.046 \pm 0.005 a$ & $117.1 \pm 4.0 a^{* *}$ \\
\hline \multirow[t]{2}{*}{ VC865 } & WW & $0.20 \pm 0.07 B$ & $19.49 \pm 1.4 \mathrm{~A}$ & $0.470 \pm 0.07 \mathrm{~A}$ & $5.81 \pm 0.52 \mathrm{~A}$ & $0.071 \pm 0.006 \mathrm{~A}$ & $44.5 \pm 7.4 \mathrm{~A}$ \\
\hline & WD & $1.40 \pm 0.12 c^{* *}$ & $8.01 \pm 0.59 b^{* *}$ & $0.077 \pm 0.01 a^{* *}$ & $1.39 \pm 0.06 a^{* *}$ & $0.041 \pm 0.003 a$ & $106.0 \pm 5.6 a^{* *}$ \\
\hline
\end{tabular}


Tab. 2 - Potential quantum yield of PSII $\left(F_{v} / F_{m}\right)$, the ratio between electron transport rate and photosynthesis (ETR/A), coefficient photochemical quenching (qL), effective quantum yield of photochemical energy conversion in PSII ( $\left.{ }_{\|}\right)$and the quantum yield of non-regulated energy $\left(\mathrm{Y}_{\mathrm{NPQ}}\right)$ in Eucalyptus clones grown in well-watered (WW) or water deficit (WD) conditions. Data are presented as the mean $\pm \operatorname{SE}(n=4)$. Capital letters compare different clones under well-watered (WW) treatment, and lower letters compare different clones under water deficit (WD) treatment using the Scott-Knott clustering $(p<0.05)$. Asterisk compares WW and WD treatments within each clone. $(* *): p<0.01 ;\left(^{*}\right): p<0.05$

\begin{tabular}{|c|c|c|c|c|c|c|}
\hline Clone & Treat & $\mathrm{F}_{\mathrm{v}} / \mathrm{F}_{\mathrm{m}}$ & $\mathrm{ETR} / A$ & $\mathrm{qL}$ & $Y_{\|}$ & $\mathrm{Y}_{\mathrm{NPQ}}$ \\
\hline \multirow[t]{2}{*}{1528} & WW & $0.83 \pm 0.001 \mathrm{~B}$ & $0.015 \pm 0.002 \mathrm{~A}$ & $0.087 \pm 0.013 \mathrm{~A}$ & $0.149 \pm 0.012 \mathrm{~A}$ & $0.49 \pm 0.02 \mathrm{~A}$ \\
\hline & WD & $0.83 \pm 0.001 \mathrm{a}$ & $0.006 \pm 0.001 a^{* *}$ & $0.078 \pm 0.008 \mathrm{a}$ & $0.107 \pm 0.009 b^{* *}$ & $0.62 \pm 0.01 a^{* *}$ \\
\hline \multirow[t]{2}{*}{1641} & WW & $0.84 \pm 0.002 \mathrm{~A}$ & $0.009 \pm 0.002 \mathrm{~B}$ & $0.051 \pm 0.007 \mathrm{~B}$ & $0.106 \pm 0.009 \mathrm{~B}$ & $0.48 \pm 0.04 \mathrm{~A}$ \\
\hline & WD & $0.84 \pm 0.003 a$ & $0.005 \pm 0.000 a^{* *}$ & $0.069 \pm 0.007 a$ & $0.107 \pm 0.010 \mathrm{~b}$ & $0.59 \pm 0.01 a^{*}$ \\
\hline \multirow[t]{2}{*}{3367} & WW & $0.83 \pm 0.007 \mathrm{~B}$ & $0.012 \pm 0.002 \mathrm{~B}$ & $0.073 \pm 0.009 \mathrm{~A}$ & $0.121 \pm 0.011 \mathrm{~B}$ & $0.54 \pm 0.01 \mathrm{~A}$ \\
\hline & WD & $0.83 \pm 0.002 \mathrm{a}$ & $0.006 \pm 0.000 a^{* *}$ & $0.063 \pm 0.002 \mathrm{a}$ & $0.110 \pm 0.005 b$ & $0.54 \pm 0.03 a$ \\
\hline \multirow{2}{*}{ gg157 } & WW & $0.84 \pm 0.003 \mathrm{~A}$ & $0.013 \pm 0.001 \mathrm{~A}$ & $0.060 \pm 0.006 \mathrm{~B}$ & $0.136 \pm 0.005 \mathrm{~A}$ & $0.42 \pm 0.02 \mathrm{~A}$ \\
\hline & WD & $0.83 \pm 0.004 a^{*}$ & $0.005 \pm 0.001 a^{* *}$ & $0.054 \pm 0.011 \mathrm{a}$ & $0.082 \pm 0.014 b^{* *}$ & $0.55 \pm 0.03 a^{* *}$ \\
\hline \multirow[t]{2}{*}{ i042 } & WW & $0.83 \pm 0.002 \mathrm{~B}$ & $0.018 \pm 0.001 \mathrm{~A}$ & $0.085 \pm 0.008 \mathrm{~A}$ & $0.159 \pm 0.014 \mathrm{~A}$ & $0.46 \pm 0.03 \mathrm{~A}$ \\
\hline & WD & $0.83 \pm 0.004 a$ & $0.004 \pm 0.000 a^{* *}$ & $0.049 \pm 0.001 a^{* *}$ & $0.076 \pm 0.003 b^{* *}$ & $0.59 \pm 0.02 a^{* *}$ \\
\hline \multirow[t]{2}{*}{ i144 } & WW & $0.83 \pm 0.004 \mathrm{~B}$ & $0.014 \pm 0.001 \mathrm{~A}$ & $0.044 \pm 0.003 \mathrm{~B}$ & $0.096 \pm 0.005 \mathrm{~B}$ & $0.44 \pm 0.03 \mathrm{~A}$ \\
\hline & WD & $0.84 \pm 0.001 \mathrm{a}$ & $0.010 \pm 0.001 a^{* *}$ & $0.080 \pm 0.005 a^{* *}$ & $0.153 \pm 0.020 a^{* *}$ & $0.47 \pm 0.07 a$ \\
\hline \multirow[t]{2}{*}{ i182 } & WW & $0.83 \pm 0.002 \mathrm{~B}$ & $0.015 \pm 0.001 \mathrm{~A}$ & $0.062 \pm 0.007 \mathrm{~B}$ & $0.116 \pm 0.010 \mathrm{~B}$ & $0.48 \pm 0.04 \mathrm{~A}$ \\
\hline & WD & $0.83 \pm 0.001 \mathrm{a}$ & $0.006 \pm 0.002 a^{* *}$ & $0.075 \pm 0.005 a$ & $0.113 \pm 0.007 \mathrm{~b}$ & $0.58 \pm 0.02 a^{*}$ \\
\hline \multirow[t]{2}{*}{ i224 } & WW & $0.83 \pm 0.003 \mathrm{~B}$ & $0.011 \pm 0.002 \mathrm{~B}$ & $0.062 \pm 0.010 \mathrm{~B}$ & $0.121 \pm 0.010 \mathrm{~B}$ & $0.45 \pm 0.04 \mathrm{~A}$ \\
\hline & WD & $0.83 \pm 0.004 a$ & $0.007 \pm 0.002 a^{*}$ & $0.057 \pm 0.007 a$ & $0.104 \pm 0.017 b$ & $0.53 \pm 0.03 a$ \\
\hline \multirow[t]{2}{*}{ PL40 } & WW & $0.84 \pm 0.001 \mathrm{~A}$ & $0.011 \pm 0.002 \mathrm{~B}$ & $0.058 \pm 0.007 \mathrm{~B}$ & $0.118 \pm 0.008 \mathrm{~B}$ & $0.48 \pm 0.05 \mathrm{~A}$ \\
\hline & WD & $0.83 \pm 0.001 \mathrm{a}$ & $0.004 \pm 0.001 a^{* *}$ & $0.059 \pm 0.006 a$ & $0.107 \pm 0.012 b$ & $0.52 \pm 0.03 a$ \\
\hline \multirow[t]{2}{*}{ VC865 } & WW & $0.83 \pm 0.003 B$ & $0.014 \pm 0.002 \mathrm{~A}$ & $0.072 \pm 0.007 \mathrm{~A}$ & $0.117 \pm 0.012 \mathrm{~B}$ & $0.55 \pm 0.02 \mathrm{~A}$ \\
\hline & WD & $0.83 \pm 0.007 \mathrm{a}$ & $0.005 \pm 0.001 a^{* *}$ & $0.067 \pm 0.014 a$ & $0.100 \pm 0.013 b$ & $0.56 \pm 0.02 \mathrm{a}$ \\
\hline
\end{tabular}

leaves of plants subjected to drought- and i144 $\left(35 \%, 50.5 \mu \mathrm{g} \mathrm{cm}^{-2}\right)$ when subjected tions was observed in clones i182 ( $9.89 \mu \mathrm{g}$ stress, especially in clones i224 (153\%, 18 to WD ( $\mathrm{p}<0.05-$ Tab. 3 ). The greatest inpmol mg-1 $)$, i144 $\left(76 \%, 10.7 \mathrm{pmol} \mathrm{mg}^{-1}\right)$ and crement of chlorophyll $b$ concentration in i042 (74\%, $\left.8.1 \mathrm{pmol} \mathrm{mg}^{-1}\right)$, compared to WW WD plants was observed in clone $1144(39 \%$, plants ( $\mathrm{p}<0.05)$. Similarly, chlorophyll a $\left.17.4 \mu \mathrm{g} \mathrm{cm} \mathrm{cm}^{-2}\right), \mathrm{i} 182\left(28 \%, 15.7 \mu \mathrm{g} \mathrm{cm} \mathrm{cm}^{-2}\right)$ and concentration increased in most of the $1641\left(28 \%, 18.5 \mu \mathrm{g} \mathrm{cm} \mathrm{cm}^{-2}\right)$. Significant inclones, especially in $\mathrm{i} 182\left(37 \%, 48.7 \mu \mathrm{g} \mathrm{cm} \mathrm{cm}^{-2}\right)$ creases in the total carotenoid concentra$\left.\mathrm{cm}^{-2}\right)$, vc865 (10.52 $\left.\mu \mathrm{g} \mathrm{cm}^{-2}\right)$ and $1528(11.05$ $\mu \mathrm{g} \mathrm{cm}^{-2}$ ), on average, $25 \%$ in plants subjected to WD compared to WW ( $p<0.05-$ Tab. 3). The carbon isotope discrimination $\left(\Delta^{13} C\right)$ was significantly reduced in clone i042 (17.99\%) and i182 (18.57\%) subjected

Tab. 3 - Abscisic acid content (ABA, pmol mg-1 FW), chlorophyll $a\left(\mathrm{Chl} a, \mu \mathrm{g} \mathrm{cm}^{-2}\right)$, chlorophyll $b\left(\mathrm{Chl} b\right.$, $\left.\mu \mathrm{g} \mathrm{cm}^{-2}\right)$, total carotenoids (Carot, $\mu \mathrm{g} \mathrm{cm}^{-2}$ ) and isotopic discrimination $\left(\triangle^{13} \mathrm{C}\right.$, \%) in Eucalyptus clones grown in well-watered (WW) or water deficit (WD) conditions. Data are presented as the mean \pm SE $(n=4)$. Capital letters compare different clones under well-watered $(W W)$ treatment, and lower letters compare different clones under water deficit (WD) treatment using the Scott-Knott clustering $(p<0.05)$. Asterisk compares WW and WD treatments within each clone. $(* *): p<0.01 ;(*): p<0.05$.

\begin{tabular}{|c|c|c|c|c|c|c|}
\hline Clone & Treat & ABA & Chl $a$ & Chl $b$ & Carot & $\Delta^{13} C$ \\
\hline \multirow[t]{2}{*}{1528} & WW & $6.2 \pm 1.0 \mathrm{C}$ & $44.1 \pm 2.4 \mathrm{~A}$ & $15.5 \pm 0.58 \mathrm{~A}$ & $10.05 \pm 0.50 \mathrm{~A}$ & $19.19 \pm 1.06 \mathrm{~B}$ \\
\hline & WD & $8.7 \pm 0.6 d$ & $51.8 \pm 4.8 a^{*}$ & $19.7 \pm 2.62 a^{* *}$ & $11.04 \pm 0.63 a$ & $17.69 \pm 0.64 b$ \\
\hline \multirow[t]{2}{*}{1641} & WW & $13.3 \pm 1.1 \mathrm{~A}$ & $42.0 \pm 1.8 \mathrm{~A}$ & $14.4 \pm 0.81 \mathrm{~A}$ & $8.16 \pm 0.92 \mathrm{~A}$ & $17.64 \pm 0.97 B$ \\
\hline & WD & $13.7 \pm 0.5 b$ & $52.8 \pm 1.3 a^{* *}$ & $18.5 \pm 0.90 a^{* *}$ & $10.07 \pm 0.92 a^{*}$ & $17.80 \pm 0.34 b$ \\
\hline \multirow[t]{2}{*}{3367} & WW & $10.9 \pm 1.6 \mathrm{~B}$ & $39.3 \pm 4.5 \mathrm{~A}$ & $13.0 \pm 1.17 \mathrm{~A}$ & $8.62 \pm 0.80 \mathrm{~A}$ & $19.58 \pm 0.75 \mathrm{~A}$ \\
\hline & WD & $11.3 \pm 1.8 \mathrm{c}$ & $48.5 \pm 2.7 a^{*}$ & $16.6 \pm 1.02 b^{* *}$ & $9.93 \pm 0.63 a$ & $17.52 \pm 0.24 b^{*}$ \\
\hline \multirow[t]{2}{*}{ gg157 } & WW & $9.0 \pm 0.2 \mathrm{~B}$ & $41.0 \pm 2.2 \mathrm{~A}$ & $13.1 \pm 0.63 \mathrm{~A}$ & $9.11 \pm 0.50 \mathrm{~A}$ & $20.16 \pm 0.30 \mathrm{~A}$ \\
\hline & WD & $15.7 \pm 2.3 b^{* *}$ & $46.1 \pm 2.9 \mathrm{a}$ & $15.0 \pm 0.86 b$ & $9.68 \pm 0.44 a$ & $18.41 \pm 0.40 b^{*}$ \\
\hline \multirow[t]{2}{*}{ i042 } & WW & $4.7 \pm 0.5 \mathrm{C}$ & $39.3 \pm 0.9 \mathrm{~A}$ & $13.6 \pm 0.36 \mathrm{~A}$ & $8.92 \pm 0.26 \mathrm{~A}$ & $20.85 \pm 0.43 \mathrm{~A}$ \\
\hline & WD & $8.1 \pm 1.5 \mathrm{~d}^{*}$ & $49.6 \pm 2.7 a^{* *}$ & $16.9 \pm 0.86 b^{*}$ & $10.51 \pm 0.93 a$ & $17.99 \pm 0.45 b^{* *}$ \\
\hline \multirow[t]{2}{*}{ ¡144 } & WW & $5.4 \pm 1.1 \mathrm{C}$ & $37.5 \pm 0.6 \mathrm{~A}$ & $12.5 \pm 0.22 \mathrm{~A}$ & $8.41 \pm 0.22 \mathrm{~A}$ & $20.72 \pm 0.50 \mathrm{~A}$ \\
\hline & WD & $10.7 \pm 0.6 c^{* *}$ & $50.5 \pm 1.5 a^{* *}$ & $17.4 \pm 0.36 a^{* *}$ & $10.22 \pm 0.64 a$ & $20.17 \pm 0.04 a$ \\
\hline \multirow[t]{2}{*}{ i182 } & WW & $5.8 \pm 0.8 \mathrm{C}$ & $35.5 \pm 1.1 \mathrm{~A}$ & $12.2 \pm 0.09 \mathrm{~A}$ & $7.57 \pm 0.36 \mathrm{~A}$ & $21.47 \pm 0.24 \mathrm{~A}$ \\
\hline & WD & $7.7 \pm 0.9 d$ & $48.7 \pm 0.9 a$ & $15.7 \pm 0.47 b^{*}$ & $9.89 \pm 0.27 a^{*}$ & $18.57 \pm 0.19 b^{* *}$ \\
\hline \multirow[t]{2}{*}{ ¡224 } & WW & $6.2 \pm 1.2 \mathrm{C}$ & $45.8 \pm 3.9 \mathrm{~A}$ & $15.6 \pm 1.31 \mathrm{~A}$ & $10.01 \pm 0.73 \mathrm{~A}$ & $19.49 \pm 0.60 \mathrm{~B}$ \\
\hline & WD & $18.0 \pm 1.0 a^{* *}$ & $49.6 \pm 5.1 a^{*}$ & $17.6 \pm 0.89 a$ & $10.17 \pm 1.44 a$ & $18.07 \pm 0.48 b$ \\
\hline \multirow[t]{2}{*}{ PL40 } & WW & $6.8 \pm 0.3 \mathrm{C}$ & $40.4 \pm 0.4 \mathrm{~A}$ & $14.0 \pm 0.45 \mathrm{~A}$ & $8.76 \pm 0.21 \mathrm{~A}$ & $19.12 \pm 0.28 \mathrm{~B}$ \\
\hline & WD & $10.2 \pm 0.7 c^{*}$ & $48.1 \pm 0.8 a^{*}$ & $16.2 \pm 0.72 b$ & $10.29 \pm 0.47 a$ & $17.50 \pm 0.57 b$ \\
\hline \multirow[t]{2}{*}{ VC865 } & WW & $7.4 \pm 1.1 \mathrm{C}$ & $38.1 \pm 2.3 \mathrm{~A}$ & $12.6 \pm 0.62 \mathrm{~A}$ & $8.51 \pm 0.36 \mathrm{~A}$ & $19.47 \pm 1.17 \mathrm{~B}$ \\
\hline & WD & $12.0 \pm 0.2 c^{* *}$ & $46.5 \pm 1.3 a^{*}$ & $15.0 \pm 0.69 b$ & $10.52 \pm 0.25 a^{*}$ & $17.48 \pm 0.51 \mathrm{~b}^{*}$ \\
\hline
\end{tabular}


Tab. 4 - Height $\left(\mathrm{cm}\right.$ plant $\left.\mathrm{t}^{-1}\right)$, stem diameter $\left(\mathrm{mm}\right.$ plant $\left.{ }^{-1}\right)$, shoot dry matter (SDM, g plant $\left.{ }^{-1}\right)$, root dry matter (RDM, g plant $\left.\mathrm{f}^{-1}\right)$, total dry matter (TDM, g plant ${ }^{-1}$ ) and the ratio RDM/TDM in Eucalyptus clones grown in well-watered (WW) or water deficit (WD) conditions. Data are presented as the mean \pm SE $(n=4)$. Capital letters compare different clones under well-watered $(W W)$ treatment, and lower letters compare different clones under water deficit (WD) treatment using the Scott-Knott clustering ( $p<0.05)$. Asterisk com pares WW and WD treatments within each clone. $(* *): p<0.01 ;\left(^{*}\right): p<0.05$.

\begin{tabular}{|c|c|c|c|c|c|c|c|}
\hline Clone & Treat & Height & Stem diameter & SDM & RDM & TDM & RDM/TDM \\
\hline \multirow[t]{2}{*}{1528} & WW & $39.38 \pm 4.17 \mathrm{C}$ & $5.77 \pm 0.48 \mathrm{~A}$ & $6.46 \pm 0.90 \mathrm{~B}$ & $2.48 \pm 0.18 \mathrm{~A}$ & $8.94 \pm 1.07 \mathrm{~B}$ & $0.28 \pm 0.02 \mathrm{~A}$ \\
\hline & WD & $29.25 \pm 3.85 c^{*}$ & $4.42 \pm 0.55 a^{* *}$ & $4.29 \pm 0.39 b$ & $2.67 \pm 0.20 a$ & $6.96 \pm 0.58 b$ & $0.38 \pm 0.01 a^{* *}$ \\
\hline \multirow[t]{2}{*}{1641} & WW & $60.63 \pm 1.71 \mathrm{~A}$ & $6.12 \pm 0.45 \mathrm{~A}$ & $12.73 \pm 1.42 \mathrm{~A}$ & $3.56 \pm 0.55 \mathrm{~A}$ & $16.29 \pm 1.93 \mathrm{~A}$ & $0.22 \pm 0.01 \mathrm{C}$ \\
\hline & WD & $49.31 \pm 1.37 \mathrm{a}^{*}$ & $4.38 \pm 0.19 a^{* *}$ & $6.52 \pm 0.72 a^{* *}$ & $2.40 \pm 0.21 a^{*}$ & $8.93 \pm 0.90 a^{* *}$ & $0.27 \pm 0.01 b^{* *}$ \\
\hline \multirow[t]{2}{*}{3367} & WW & $48.00 \pm 5.05 B$ & $5.31 \pm 0.16 \mathrm{~A}$ & $8.44 \pm 0.71 B$ & $2.59 \pm 0.30 \mathrm{~A}$ & $11.03 \pm 0.83 B$ & $0.24 \pm 0.02 \mathrm{~B}$ \\
\hline & WD & $37.25 \pm 4.54 c^{*}$ & $3.80 \pm 0.16 a^{* *}$ & $4.46 \pm 0.43 b^{*}$ & $1.51 \pm 0.05 b^{*}$ & $5.97 \pm 0.47 b^{*}$ & $0.26 \pm 0.01 b$ \\
\hline \multirow[t]{2}{*}{ gg157 } & WW & $42.50 \pm 1.17 \mathrm{C}$ & $5.04 \pm 0.23 \mathrm{~A}$ & $6.42 \pm 0.35 B$ & $1.55 \pm 0.13 B$ & $7.96 \pm 0.48 B$ & $0.19 \pm 0.01 \mathrm{C}$ \\
\hline & WD & $33.31 \pm 1.49 c^{*}$ & $3.87 \pm 0.25 a^{*}$ & $3.18 \pm 0.25 b^{*}$ & $1.67 \pm 0.12 b$ & $4.84 \pm 0.33 b$ & $0.35 \pm 0.02 a^{* *}$ \\
\hline \multirow[t]{2}{*}{ i042 } & WW & $58.00 \pm 3.92 \mathrm{~A}$ & $5.65 \pm 0.41 \mathrm{~A}$ & $9.09 \pm 1.25 B$ & $2.69 \pm 0.23 \mathrm{~A}$ & $11.78 \pm 1.45 B$ & $0.23 \pm 0.01 \mathrm{~B}$ \\
\hline & WD & $42.81 \pm 6.55 b^{* *}$ & $4.69 \pm 0.29 a$ & $4.30 \pm 0.78 b^{* *}$ & $1.83 \pm 0.32 b$ & $6.13 \pm 1.08 b^{* *}$ & $0.30 \pm 0.02 b^{* *}$ \\
\hline \multirow[t]{2}{*}{ ¡144 } & WW & $39.00 \pm 1.21 \mathrm{C}$ & $4.69 \pm 0.24 \mathrm{~A}$ & $5.66 \pm 0.32 B$ & $1.87 \pm 0.05 B$ & $7.53 \pm 0.33 B$ & $0.25 \pm 0.01 \mathrm{~B}$ \\
\hline & WD & $30.42 \pm 1.72 c^{*}$ & $4.01 \pm 0.51 \mathrm{a}$ & $3.36 \pm 0.77 b$ & $1.65 \pm 0.24 b$ & $5.01 \pm 1.02 b$ & $0.34 \pm 0.02 a^{* *}$ \\
\hline \multirow[t]{2}{*}{ i182 } & WW & $48.69 \pm 1.57 \mathrm{~B}$ & $5.94 \pm 0.23 \mathrm{~A}$ & $11.73 \pm 1.04 \mathrm{~A}$ & $2.72 \pm 0.19 \mathrm{~A}$ & $14.45 \pm 1.23 \mathrm{~A}$ & $0.19 \pm 0.01 \mathrm{C}$ \\
\hline & WD & $37.19 \pm 2.37 c^{*}$ & $4.44 \pm 0.06 a^{* *}$ & $5.97 \pm 0.79 a^{* *}$ & $2.33 \pm 0.34 a$ & $8.30 \pm 1.09 a^{* *}$ & $0.28 \pm 0.01 b^{* *}$ \\
\hline \multirow[t]{2}{*}{$\mathrm{i} 224$} & WW & $48.63 \pm 3.31 \mathrm{~B}$ & $5.48 \pm 0.28 \mathrm{~A}$ & $8.04 \pm 1.58 B$ & $2.49 \pm 0.46 \mathrm{~A}$ & $10.54 \pm 2.01 \mathrm{~B}$ & $0.24 \pm 0.01 \mathrm{~B}$ \\
\hline & WD & $37.69 \pm 0.95 c^{*}$ & $4.55 \pm 0.30 a$ & $5.51 \pm 0.87 b$ & $2.24 \pm 0.48 a$ & $7.75 \pm 1.32 b$ & $0.28 \pm 0.02 b^{* *}$ \\
\hline \multirow[t]{2}{*}{ PL40 } & WW & $52.75 \pm 3.55 \mathrm{~A}$ & $5.24 \pm 0.74 \mathrm{~A}$ & $13.33 \pm 2.57 \mathrm{~A}$ & $3.37 \pm 0.63 \mathrm{~A}$ & $16.70 \pm 3.19 \mathrm{~A}$ & $0.20 \pm 0.01 \mathrm{C}$ \\
\hline & WD & $43.13 \pm 4.71 b^{*}$ & $4.06 \pm 0.26 a^{*}$ & $4.26 \pm 0.44 b^{* *}$ & $1.65 \pm 0.26 b^{* *}$ & $5.91 \pm 0.69 b^{* *}$ & $0.28 \pm 0.02 b^{* *}$ \\
\hline \multirow[t]{2}{*}{ VC865 } & WW & $54.38 \pm 1.43 \mathrm{~A}$ & $5.83 \pm 0.39 \mathrm{~A}$ & $11.79 \pm 2.52 \mathrm{~A}$ & $2.81 \pm 0.42 \mathrm{~A}$ & $14.60 \pm 2.93 \mathrm{~A}$ & $0.20 \pm 0.01 \mathrm{C}$ \\
\hline & WD & $54.50 \pm 2.25 a$ & $5.51 \pm 0.21 a$ & $9.12 \pm 0.78 a$ & $3.36 \pm 0.35 a$ & $12.48 \pm 1.03 a$ & $0.27 \pm 0.02 b^{* *}$ \\
\hline
\end{tabular}

to $\mathrm{WD}$, in $14 \%$ when compared to $\mathrm{WW}$ plants $(p<0.05)$. No differences were found in carbon isotope discrimination in control plants of clones 1528, 1641, i144, i224, and PL040 compared to the droughtstressed plants.

\section{Growth and SDM/RDM ratio}

We observed for the clone PL40 a greater reduction in height $(\mathrm{H}-\mathrm{p}<0.05)$, shoot dry matter $(\mathrm{SDM}-\mathrm{p}<0.05)$ and total dry matter (TDM - p <0.05) in WD as compared to WW, with TDM reductions up to $64 \%$ (5.91 g plant $\left.^{-1}\right)$, followed by $3367(54 \%, 5.97 \mathrm{~cm}$ plant $\left.{ }^{-1}\right)$ and io $42\left(48 \%, 6.13 \mathrm{~cm}^{-1 a n t^{-1}}-\right.$ Tab. 4). The lowest reduction in SDM was observed in clone i224 $\left(5.51 \mathrm{~cm}\right.$ plant $\left.^{-1}, 32 \%\right)$ compared to WW plants (Tab. 4). Root dry matter (RDM) was not affected by WD conditions in clones 1528 and gg157. However, the lowest ratios of reduction in shoot to root dry matter (SDM/RDM - data not shown) and of the root to total dry matter ratio (RDM/TDM) were found for clone 3367 (13.2 and $8.6 \%$, respectively) under water-deficit conditions ( $<<0.05-$ Tab. 4). No significant changes in the TDM of plants of clones 1528, and i224 were detected under WD (Tab. 4), and the lowest reduction was found by $1144\left(5.01 \mathrm{~cm}^{2}\right.$ plant $\left.^{-1} ; 33 \%\right)$, a drought-tolerant genotype $(\mathrm{p}<0.05)$.

\section{PCA and hierarchical clustering}

Four principal components (PCs), based on relative values, explained $79 \%$ of the total variation in the analyzed data, and the first two PCs explained $33 \%$ and $19 \%$, respectively (Fig. 1). The first PC was characterized by high positive scores for gas ex- change traits $\left(A / C i, A, E T R / A, E T R, E, g_{s}\right.$ and Ward's linkage allowed to categorize the $Y_{I I}$ ) and negatively associated with root Eucalyptus clones into four groups based characteristics (RDM, RDM/TDM), $\Psi_{w}$ and on the relative magnitude of the measured $Y_{I I}$ (Fig. 1). The second PC had a strong posi- responses to treatment: (i) Clone i144 as retive correlation with pigments and WUE sistant and (ii) 3367 and i224 as moderately and negative correlation with $\mathrm{Ci} / \mathrm{Ca}$. Other resistant to the effects of water deficit. All variables such as $\mathrm{ABA}, \mathrm{SD}, \Delta^{13} \mathrm{C}$ had minor were characterized by the lowest reduceffects on the first two PCs evaluated (Fig. tion in gas exchange traits, indicating con1).

Analyzing all physiological and morphological evaluated traits among various genotypes and the dendrogram using tinued carbon gain (Fig. 2). Clone i144 also demonstrated no change in $\mathrm{A} / \mathrm{Ci}$ and showed the highest $A B A$ levels under drought. The other two groups character-

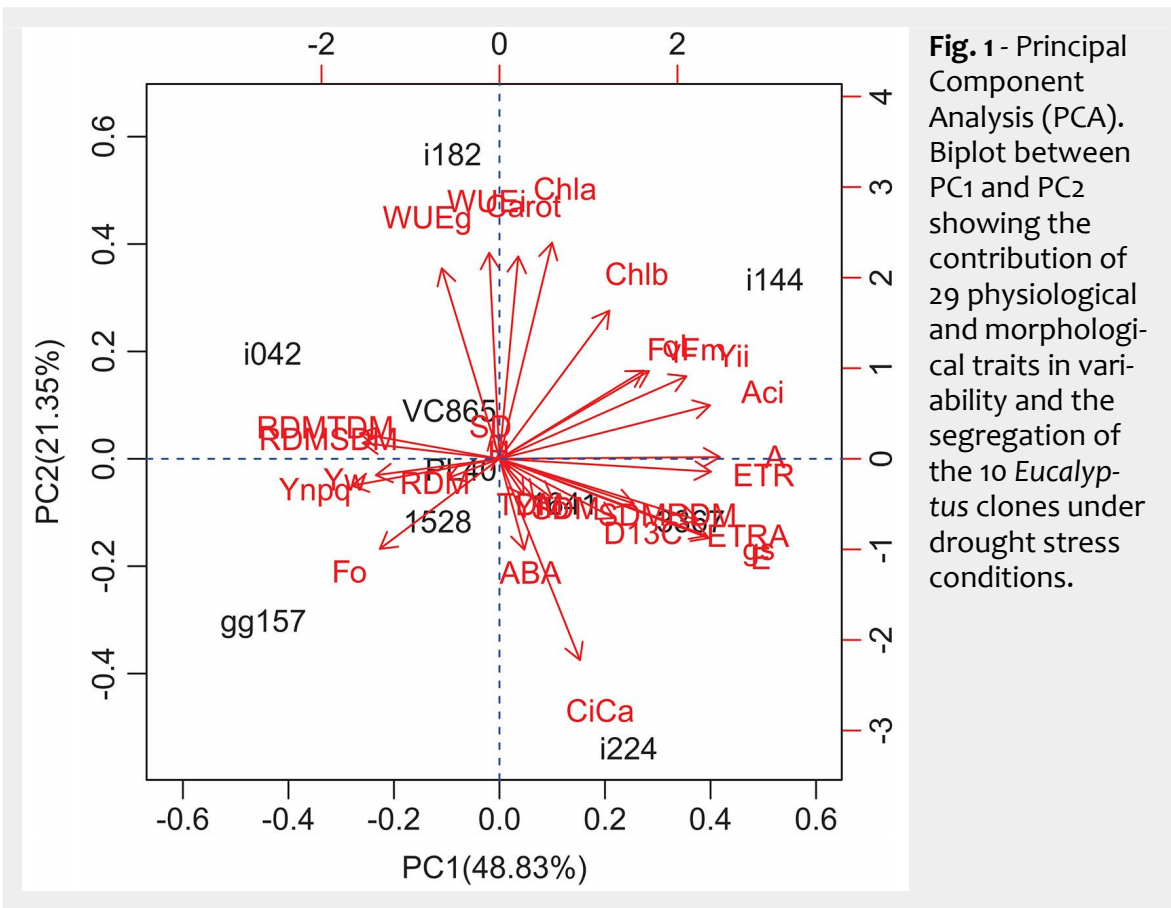


Fig. 2 - Hierarchical clustering analysis. Dendrogram of 10 Eucalyptus clones using Ward's linkage with Bray-Curtis dissimilarity measure. Data consists in relative values of 29 physiologica and morphological traits in eucalypt plants subjected to drought stress conditions.

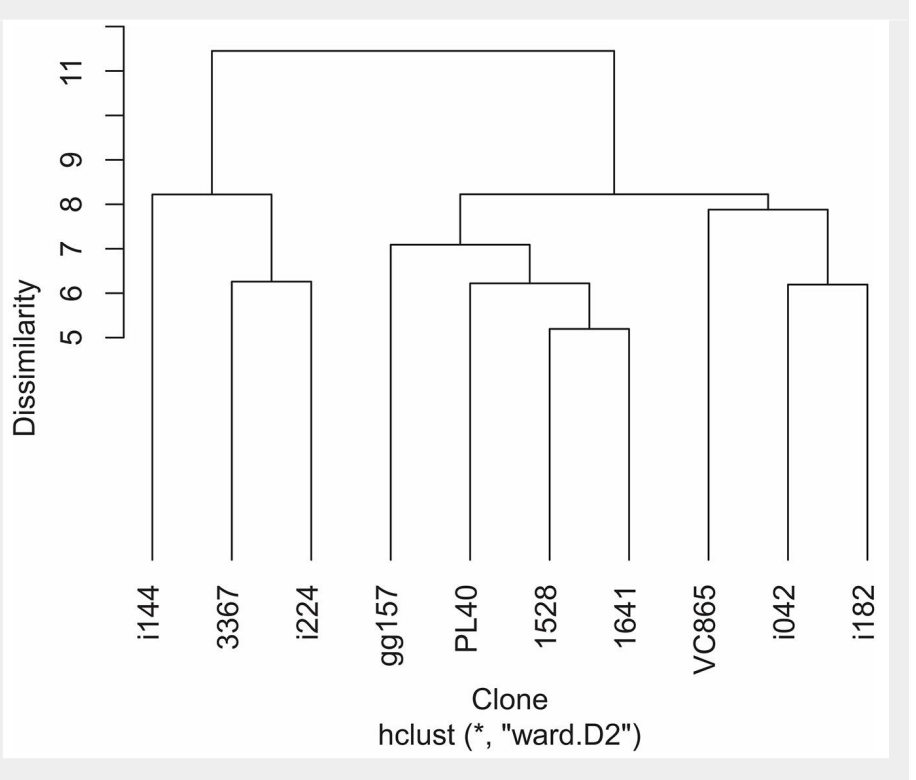

ized as follows: (iii) clones gg157, pl40, 1528 and 1641, moderately-sensitive, where 1528 was not affected in RDM, gg157 showed the highest increases in RDM and $\mathrm{Y}_{\mathrm{NPQ}}$ variables; and (iv) vc865, i182 and i042, characterized as sensitive clones to drought stress. Clones vc865 and i182 invested in shoot growth, to the detriment of the root system. Clone 1182 had an increment of WUE and reduced $\Delta^{13} \mathrm{C}$; however, these alterations were not reflected in drought-tolerance during water stress.

\section{Discussion}

Drought-tolerant genotypes had lower negative effects on gas exchange and carbon isotope composition during water stress

Photosynthesis is one of the primary processes initially affected by water restriction in plants (Chaves et al. 2009), mainly in isohydric species (Tardieu \& Simonneau 1998). The imposition of water deficit clones resulted in reductions in $A, C_{i}, E$ and $g_{s}$ that were more pronounced in clones io42 (sensitive), gg157 and i182, considered within the context of our study as moderately sensitive or sensitive to drought stress.

The reduction in stomatal conductance observed here may be partially mediated by higher ABA concentrations (Schachtman \& Goodger 2008) and osmotic adjustment (Merchant et al. 2006, Arndt et al. 2008), leading to increased water-use efficiency (Parry et al. 2005) and avoiding unnecessary water loss. Clones i042, i144 and i224 contained higher ABA levels in the leaves and greatly reduced stomatal conductance; nevertheless, no significant correlation was obtained between $A B A$ and $g_{s}$ (data not shown). For genotypes 1528, gg157, 3367 and i182 however, there were no differences in ABA leaf concentrations; however, stomatal conductance was greatly reduced under drought. Taken together, these results suggest that stomatal closure in these genotypes is not mediated by
$A B A$. Alternatively, ABA regulation of stomatal aperture may be masked by rapid metabolism during the first stages of drought, therefore it does not accumulate in the leaves (Jiang \& Hartung 2008). Some studies have shown the relationship of higher ABA accumulation and drought tolerance in Eucalyptus genotypes (Valdés et al. 2013, Martins et al. 2018). In this experiment, clone i144 had an approximate $40 \%$ increase in ABA concentration, which possibly contributed to the increase $(\sim 156 \%)$ in water use efficiency (WUE - Tab. 1), confirming the drought-tolerant characteristics of this genotype.

Increase in water-use efficiency is thought to be an important property that contributes to the resilience and growth of genotypes under drought conditions ( $\mathrm{Li}$ et al. 2009). Our results suggest that while significant variability exists in the parameters measured in this study, it is clear that factors governing leaf gas exchange did not delineate the genotypes under the various treatment conditions. Surrogate measures of leaf gas exchange, including carbon isotope abundance, may offer significant insight into the tolerance of individual genotypes to the effects of water deficit (Macfarlane et al. 2004, Schulze et al. 2014). Nevertheless, the tolerant genotype (i144), and other clones considered to be moderately sensitive genotypes (1528, 1641, i224, and PL040) did not show differences in leaf $\Delta^{13} C$, suggesting an absence of pattern to this trait to drought tolerance selection in early stages of Eucalyptus growth. One possible explanation for this may be that carbon acquisition was not of a sufficient magnitude under the stress conditions; therefore, a change in isotope abundance was not observed. This represents a significant limitation in the use of $\Delta^{13} \mathrm{C}$ as an indicator of plant stress tolerance and, at the very least, needs to be considered in the context of net carbon assimilation.
Non-stomatal limitations to photosynthesis were observed among Eucalyptus clones

Differential limitations to photosynthesis were observed among clones under the effects of water deficit. In long-term and moderate stress, the reduction of $g_{s}$ can induce a decrease in $\mathrm{CO}_{2}$ concentration at the carboxylation sites of RuBisCO (Flexas et al. 2004, 2007), with consequent stomatal limitation that reduces photosynthetic rates. However, in this study, internal $\mathrm{CO}_{2}$ concentrations $\left(\mathrm{C}_{\mathrm{i}}\right)$ and the corresponding $A / C_{i}$ were not proportionately reduced for most clones (1528, gg157, i042, i182 - moderately-sensitive or sensitive to drought), suggesting that, under the conditions tested here, changes or damage to photosynthetic metabolism (Lawlor \& Tezara 2009) resulted in non-stomatal limitation of $A$.

RUBP regeneration capacity, a non-stomatal limitation, is limited by drought, possibly due to reduction in the electron transport rate and, consequently, in NADPH (Flexas et al. 2004). These authors found that the decrease in photochemical efficiency was evidenced by a high reduction in the electron transport rate (ETR), accompanied by a reduction in photochemical quenching ( $\mathrm{qL})$, as observed in the present study, especially in the droughtsensitive clone i042 under water-deficit conditions. Decreases in A and ETR are generally proportional, suggesting a strong link between the photosynthetic processes (Foyer et al. 1990). Alternatively, a decrease in $A$ and maintenance of ETR, leading to an increased ETR/A ratio, suggest that water deficit causes no inhibition of photochemical reactions (Singh \& Raja Reddy 2011). In the present study, for almost all genotypes, the ETR/A and $A / C_{i}$ ratios decreased, especially for clones i042 and i182, characterized here as sensitive to water deficit. These responses suggest that the reduction in the electron transport rate was as high as the drop in the photosynthetic rate during water deficit. Although the possible biological limitations were not measured in this study, drastic changes in the above-mentioned processes suggest predominance of non-stomatal limitations in these genotypes.

Conversely, in the drought-tolerant genotype (i144), slight reductions in ETR and increases in $\mathrm{qL}$, associated with unchanged $A / C_{i}$ and $E T R / A$ ratios, suggest a decrease in $A$ by stomatal limitations. Clones 3367 and i224 were similar to i144 in terms of these characteristics and were characterized as moderately tolerant genotypes. The results suggest that these genotypes have a greater potential for post-stress recovery, because the photosynthetic apparatus remains functional during water-deficit periods. Alternatively, higher photosynthetic rates during the early drought stages also increase plant survival and dry matter accumulation (Parry et al. 2005); in genotypes with higher $A$ under water-deficit condi- 
tions, photoinhibition of photosynthesis is reduced (Singh \& Raja Reddy 2011).

Excess energy dissipation may also result from the accumulation of reducing power (NADPH) and ATP produced in the photochemical phase. This is subsequently released in the form of thermal energy, measured by NPQ. The dissipation of absorbed energy through thermal dissipation (NPQ) increased most in clones 1528 and i182. In these genotypes, the thermal dissipation may have been important to avoid photoinhibition. This is because dissipation of excess energy, either photochemically or non-photochemically (e.g., thermal dissipation) controls excess excitation energy and electron fluxes to $\mathrm{O}_{2}$, that can prevent photooxidative damage (Murata et al. 2012) and consequently photoinhibition (Roach \& Krieger-Liszkay 2014).

Factors governing gas exchange and chlorophyll a fluorescence undoubtedly played a major role in the stress response of individual clones, highlighting the need for integrated measures of plant scale physiological measurements to be part of the pre-breeding toolbox. We also showed that non-invasive parameters reflecting non-stomatal factors such as ETR/A may be used as part of the suite of tools for assessment of performance under drought conditions.

\section{Genotype growth and classification}

Clone i144, considered drought-tolerant in the field and confirmed in this study as tolerant during seedling stage and greenhouse controlled-conditions, performed well compared to other clones under water stress. This performance may have been the result of minor changes in $A, g_{s}, C_{i} C_{a}$, and minor damage to the photosynthetic apparatus (lower ETR/A) as well as the lower SDM/RDM ratio. The lower SDM/ RDM ratio indicates expansion of the root system at the expense of shoot growth that allows for better recovery after water deficit (Mokotedi et al. 2010). To increase water uptake, many plants increase root growth, laterally or in terms of depth. In Eucalyptus species, the difference in drought tolerance was attributed to differences in root depth (Mokotedi et al. 2010) and the hydraulic conductivity of the root system (Silva et al. 2004). Therefore, the above-mentioned characteristics may have led to a greater increase in WUE and continued growth despite the effects of water deficit. The performance of the clones 3367 and i224 was similar to that of the tolerant clone (i144) in terms of most characteristics evaluated, and were characterized as moderately tolerant to drought.

The largest reductions in growth and photosynthetic rate observed for clone io42 reinforce the classification of its susceptibility to water deficit and drought under field conditions (Nunes et al. 2016). It is noteworthy that although clones i042, vc865 and 1182 were in closed groups by multivariate analysis, the last genotype was moder- ately drought-tolerant in the field. However, this potential was not expressed under greenhouse conditions in this study. According to the distribution of the dendrograms and univariate analysis of studied traits, the genotypes i224 and 3367 were classified as moderately drought-tolerant, and other genotypes were classified as moderately sensitive.

\section{Conclusions}

Assessing drought tolerance under field conditions faces significant challenges because of the scale and accessibility of leaf and root tissues, canopy and soil heterogeneity and the dynamics of soil/water/ plant interactions. Developing selection traits at the seedling stage prior to investment in field planting allows selection to take place early in the developmental cycle as well as providing a relatively consistent imposition of stress to standardize the severity of stress conditions. For the present study, it is important to emphasize that we were not able to identify a "standalone" variable characterizing drought-tolerance in the Eucalyptus clones tested. It is far more likely that a combination of such tools, including $A, E T R / A$ and SDM/RDM, will yield the most effective form of prebreeding assessment, to provide informed analysis of drought-tolerant genotypes.

Factors governing gas exchange undoubtedly played a major role in the stress response of individual clones, highlighting the need for integrated measures of plant scale gas exchange to be part of the prebreeding toolbox. We also showed that non-invasive parameters reflecting nonstomatal factors such as ETR/A may be used as part of the suite of tools for assessment of performance under drought conditions. The assessment of these traits, under the conditions imposed in this study, highlight the substantial potential for rapid assessment of physiological and chemical parameters to improve plant breeding efforts while recognizing the need to validate these tools under field conditions. Improved insight into such relations will not only inform breeding programs of the mechanistic relationships with stress tolerance, but will also provide candidate tools for use in plantation management under varying environmental and climatic conditions.

\section{List of abbreviations}

$(A)$ : net photosynthetic assimilation rate; $\left(A / C_{i}\right)$ : carboxylation efficiency; (ABA): abscisic acid; (Carot): total carotenoids; (Chla): chlorophyll $a$; (Chlb): chlorophyll b; $\left(C_{j} / C_{a}\right)$ : ratio between internal and external $\mathrm{CO}_{2}$ concentration; $(E)$ : transpiration rate; (ETR): apparent electron transport rate; $\left(F_{v} / F_{m}\right)$ : potential quantum yield of PSII; $\left(g_{s}\right)$ : stomatal conductance; (PEG): polyethylene glycol; (PSII): photosystem II; (qL): coefficient photochemical quenching; (RDM): root dry matter; (SDM): shoot dry matter; (TDM): total dry matter (TDM);
(WD): water deficit; (WUE): water use efficiency; (WW): well-watered; $\left(Y_{\|}\right)$: effective quantum yield of photochemical energy conversion in PSII; $\left(\mathrm{Y}_{\mathrm{NPQ}}\right)$ : quantum yield of non-regulated energy; $\left(\Delta^{13} C\right)$ : isotope discrimination; $\left(\Psi_{w}\right)$ : predawn water potential.

\section{Acknowledgments}

This work was supported by the Minas Gerais State Agency for Research and Development (FAPEMIG), the Coordination of Improvement of Higher Education Personnel (CAPES/PRODOC, grant number 00113/ 010-0) and the National Council for Scientific and Technological Development (CNPq/Universal, grant number 475787 / 2012-9). CM and BERH are grateful to the CAPES, CNPq and Science without borders (CSF) for scholarships. AM acknowledges the Australian Research Council Future Fellowship Scheme (FT120100200).

\section{Author contributions}

CM and NFB designed the study; CM conducted the experiments and performed the physiological measurements; BERH performed the carbon isotope composition analysis; $\mathrm{CM}, \mathrm{BERH}$ and $\mathrm{AM}$ wrote the manuscript. All authors discussed the data, read and approved the final version of the manuscript.

\section{Disclosure statement}

No potential conflict of interest was reported by the authors.

\section{References}

Arndt SK, Livesley SJ, Merchant A, Bleby TM, Grierson PF (2008). Quercitol and osmotic adaptation of field-grown Eucalyptus under seasonal drought stress. Plant, Cell and Environment 31: 915-924. - doi: 10.1111/j.1365-3040.2008.01803.x Chaves MM, Flexas J, Pinheiro C (2009). Photosynthesis under drought and salt stress: regulation mechanisms from whole plant to cell. Annals of Botany 103: 551-560. - doi: 10.1093/aob/ mcn125

Clark RB (1975). Characterization of phosphatese in intact maize roots. Journal of Agricultural and Food Chemistry 23: 458-60. - doi: 10.1021/ jf60199aoo2

Corrêa TR, Picoli EAT, Souza GA, Condé AS, Silva NM, Lopes-Mattos KLB, Resende MDV, Zauza EAV, Oda S (2017). Phenotypic markers in early selection for tolerance to dieback in Eucalyptus. Industrial Crops and Products 107: 130-138. - doi: 10.1016/j.indcrop.2017.05.032

Demmig-Adams B, Adams WW (1996). The role of xanthophyll cycle carotenoids in the protection of photosynthesis. Trends in Plant Science 1: 21-26. doi: 10.1016/S1360-1385(96)80019-7

Durgbanshi A, Arbona V, Pozo O, Miersch O, Sancho JV, Gómez-Cadenas A (2005). Simultaneous determination of multiple phytohormones in plant extracts by liquid chromatography-electrospray tandem mass spectrometry. Journal of Agricultural and Food Chemistry 53: 84378442. - doi: 10.1021/jf050884b

Farquhar GD, Richards RA (1984). Isotopic composition of plant carbon correlates with water 
use efficiency of wheat genotypes. Australian Journal of Plant Physiology 11: 539-552. - doi: 10.1071/PP9840539

Flexas J, Bota J, Loreto F, Cornic G, Sharkey TD (2004). Diffusive and metabolic limitations to photosynthesis under drought and salinity in $\mathrm{C}_{3}$ plants. Plant Biology 6: 269-279. - doi: 10.1055/s2004-820867

Flexas J, Diaz-Espejo A, Galmés J, Kaldenhoff R, Medrano H, Ribas-Carbo M (2007). Rapid variations of mesophyll conductance in response to changes in $\mathrm{CO}_{2}$ concentration around leaves. Plant, Cell and Environment 30: 1284-1298. - doi: 10.1111/j.1365-3040.2007.01700.x

Foyer C, Furbank R, Harbinson J, Horton P (1990). The mechanisms contributing to photosynthetic control of electron transport by carbon assimilation in leaves. Photosynthesis Research 25: 83-100. - doi: 10.1007/BF00035457 Genty B, Briantais J-M, Baker NR (1989). The relationship between the quantum yield of photosynthetic electron transport and quenching of chlorophyll fluorescence. Biochimica et Biophysica Acta - General Subjects 990: 87-92. doi: 10.1016/S0304-4165(89)80016-9

IBA (2019). IBA Relatório 2019 [IBA Report 2019]. Indústria Brasileira de Árvores, Pöyry Consultoria em Gestão e Negócios Ltda., Brazil, pp. 79. [in Portuguese] [online] URL: http://www.iba. org/datafiles/publicacoes/relatorios/iba-relatori oanual2019.pdf

Jiang F, Hartung W (2008). Long-distance signalling of abscisic acid ( $A B A)$ : the factors regulating the intensity of the ABA signal. Journal of Experimental Botany 59: 37-43. - doi: 10.1093/ jxb/erm127

Lawlor DW, Tezara W (2009). Causes of decreased photosynthetic rate and metabolic capacity in water-deficient leaf cells: a critical evaluation of mechanisms and integration of processes. Annals of Botany 103: 561-579. - doi: 10.1093/aob/mcn244

Li FL, Bao WK, Wu N (2009). Effects of water stress on growth, dry matter allocation and water-use efficiency of a leguminous species, Sophora davidii. Agroforestry Systems 77: 193201. - doi: 10.1007/s10457-008-9199-1

Macfarlane C, Adams MA, White D (2004). Productivity, carbon isotope discrimination and leaf traits of trees of Eucalyptus globulus Labill. in relation to water availability. Plant, Cell and Environment 27: 1515-1524. - doi: 10.1111/j.13653040.2004.01260.x

Martins GS, Freitas NC, Máximo WPF, Paiva LV (2018). Gene expression in two contrasting hybrid clones of Eucalyptus camaldulensis $\times$ Eucalyptus urophylla grown under water deficit conditions. Journal of Plant Physiology 229: 122-131. - doi: 10.1016/j.jplph.2018.07.007

Maxwell K, Johnson GN (2000). Chlorophyll fluorescence - a pratical guide. Journal of Experimental Botany 51: 659668. - doi: 10.1093/jexbot/ 51.345 .659

Medrano H, Escalona JM, Bota J, Gulías J, Flexas $J$ (2002). Regulation of photosynthesis of $C_{3}$ plants in response to progressive drought: stomatal conductance as a reference parameter. Annals of Botany 89: 895-905. - doi: 10.1093 laob/mcfo79

Merchant A, Tauz M, Arndt SK, Adams MA
(2006). Cyclitols and carbohydrates in leaves and roots of 13 Eucalyptus species suggest contrasting physiological responses to water deficit. Plant, Cell and Environment 29: 2017-2029. doi: 10.1111/j.1365-3040.2006.01577.x

Merchant A, Ladiges PY, Adams MA (2007). Quercitol links the physiology, taxonomy and evolution of 279 eucalypt species. Global Ecology and Biogeography 16: 810-819. - doi: 10.1111/ j.1466-8238.2007.00338.x

Merchant A, Arndt SK, Rowell DM, Posch S, Callister A, Tauz M, Adams MA (2010). Seasonal changes in carbohydrates, cyclitols, and water relations of 3 field grown Eucalyptus species from contrasting taxonomy on a common site. Annals of Forest Science 67: 104. - doi: 10.1051/ forest $/ 2009085$

Michel BE, Kaufmann MR (1973). The osmotic potential of polyethylene glycol 6000. Plant Physiology 51: 914-916. - doi: 10.1104/pp.51.5.914 Mokotedi MEO, Watt MP, Pammenter NW (2010). Analysis of differences in field performance of vegetatively and seed propagated Eucalyptus varieties II: vertical uprooting resistance. Southern Forests 72: 712-718. - doi: 10.29 89/20702620.2010.481131

Müller C, Silveira SFDS, Daloso DM, Mendes GC, Merchant A, Kuki KN, Oliva MA, Loureiro ME, Almeida AM (2017). Ecophysiological responses to excess iron in lowland and upland rice cultivars. Chemosphere 189: 123-133. - doi: 10.1016/j. chemosphere.2017.09.033

Murata N, Allakhverdiev SI, Nishiyama Y (2012). The mechanism of photoinhibition in vivo: Reevaluation of the roles of catalase, a-tocopherol, non-photochemical quenching, and electron transport. Biochimica et Biophysica Acta (BBA) 1817: 1127-1133. - doi: 10.1016/j.bba bio.2012.02.020

Nunes FN, Barros NF, Novais RB, Silva IR, Stape $J \mathrm{~L}$ (2016). Carbon isotope discrimination and differential drought tolerance in eucalypt clones. Scientia Forestalis 44: 895-903. [online] URL: http://www.cabdirect.org/cabdirect/abstr act/20173100565

Parry MAJ, Flexas J, Medrano H (2005). Prospects for crop production under drought: research priorities and future directions. Annals of Applied Biology 147: 211-226. - doi: 10.1111/j.17 44-7348.2005.00032.x

Pinto DS, Resende RT, Mesquita AGG, Rosado AM, Cruz CD (2014). Early selection in tests for growth traits of Eucalyptus urophylla clones test. Scientia Forestalis 42: 251-257. [online] URL: http://www.cabdirect.org/cabdirect/abstr act/20143309403

Roach T, Krieger-Liszkay AK (2014). Regulation of photosynthetic electron transport and photoinhibition. Current Protein and Peptide Science 15: 351-362. - doi: 10.2174/138920371566614 0327105143

Schachtman DP, Goodger JQD (2008). Chemical root to shoot signaling under drought. Trends in Plant Sciences 13: 281-287. - doi: 10.1016/j.t plants.2008.04.003

Schulze ED, Nicolle D, Boerner A, Lauerer M, Aas G, Schulze I (2014). Stable carbon and nitrogen isotope ratios of Eucalyptus and Acacia species along a seasonal rainfall gradient in Western Australia. Trees 28: 1125-1135. - doi: 10.1007/soo
468-014-1023-0

Silva FC, Shvaleva A, Maroco JP, Almeida MH, Chaves MM, Pereira JS (2004). Responses to water stress in two Eucalyptus globulus clones differing in drought tolerance. Tree Physiology 24 (10): 1165-1172. - doi: 10.1093/treephys/24.10. 1165

Singh SK, Raja Reddy K (2011). Regulation of photosynthesis, fluorescence, stomatal conductance and water-use efficiency of cowpea (Vigna unguiculata [L.] Walp.). under drought. Journal of Photochemistry and Photobiology B 105: 40-50. - doi: 10.1016/j.jphotobiol.2011.07.001 Stape JL, Binkley D, Ryan MG (2008). Production and carbon allocation in a clonal Eucalyptus plantation with water and nutrient manipulations. Forest Ecology and Management 255: 920-930. - doi: 10.1016/j.foreco.2007.09.085

Tardieu F, Simonneau T (1998). Variability among species of stomatal control under fluctuating soil water status and evaporative demand: modelling isohydric and anisohydric behaviours. Journal of Experimental Botany 49: 419432. - doi: 10.1093/jxb/49.Special_Issue.419

Valdés AE, Majada JP, Rodríguez A, Fernández B, Pagès $M$ (2013). Drought tolerance acquisition in Eucalyptus globulus (Labill.): a research on plant morphology, physiology and proteomics. Journal of Proteomics 79: 263-276. - doi: 10.1016 /j.jprot.2012.12.019

Warren CR, Bleby TM, Adams MA (2007). Changes in gas exchange versus leaf solutes as a mean to cope with summer drought in Eucalyptus marginata. Oecologia 154: 1-10. - doi: 10.10 07/s00442-007-0803-2

Warren CR, Aranda I, Cano FJ (2011). Responses to water stress of gas exchange and metabolites in Eucalyptus and Acacia spp. Plant, Cell and Environment 34: 1609-1629. - doi: 10.1111/j.13 65-3040.2011.02357.x

Warren CR, Aranda I, Cano FJ (2012). Metabolomics demonstrates divergent responses of two Eucalyptus species to water stress. Metabolomics 8: 186-200. - doi: 10.1007/s11306-0110299-y

Wellburn AR (1994). The spectral determination of chlorophylls $a$ and $b$, as well as total carotenoids, using various solvents with spectrophotometers of different resolution. Journal of Plant Physiology 144: 307-313. - doi: 10.1016/So1 76-1617(11)81192-2

White DA, Crombie DS, Kinal J, Battaglia M, MCgrath JF, Mendharn DS, Walker SN (2009). Managing productivity and drought risk in Eucalyptus globulus plantations in south-western Australia. Forest Ecology and Management 259 (1): 33-44. - doi: 10.1016/j.foreco.2009.09.039 Zhou Y, Huang L, Zhang Y, Shi K, Yu J, Nogués S (2007). Chill induced decrease in capacity of RuBP carboxylation and associated $\mathrm{H}_{2} \mathrm{O}_{2}$ accumulation in cucumber leaves are alleviated by grafting onto figleaf Gourd. Annals of Botany 100: 839-848. - doi: 10.1093/aob/mcm181

Zhou SX, Medlyn BE, Prentice IC (2016). Longterm water stress leads to acclimation of drought sensitivity of photosynthetic capacity in xeric but not riparian Eucalyptus species. Annals of Botany 117: 133-144. - doi: 10.1093/aob/ mcv161 